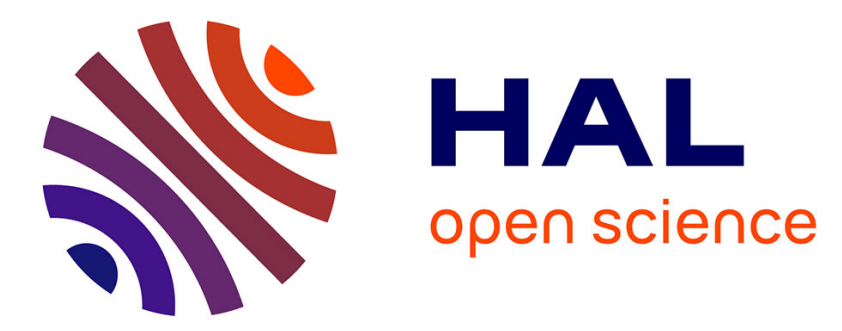

\title{
Micro/macro-scale robotic approach for middle ear surgery
}

Jae-Hun So, Brahim Tamadazte, Jérôme Szewczyk

\section{To cite this version:}

Jae-Hun So, Brahim Tamadazte, Jérôme Szewczyk. Micro/macro-scale robotic approach for middle ear surgery. IEEE Transactions on Medical Robotics and Bionics, 2020, 2 (4), pp.533 - 536. 10.1109/TMRB.2020.3032456 . hal-03053016

\section{HAL Id: hal-03053016 https://hal.science/hal-03053016}

Submitted on 10 Dec 2020

HAL is a multi-disciplinary open access archive for the deposit and dissemination of scientific research documents, whether they are published or not. The documents may come from teaching and research institutions in France or abroad, or from public or private research centers.
L'archive ouverte pluridisciplinaire $\mathbf{H A L}$, est destinée au dépôt et à la diffusion de documents scientifiques de niveau recherche, publiés ou non, émanant des établissements d'enseignement et de recherche français ou étrangers, des laboratoires publics ou privés. 
See discussions, stats, and author profiles for this publication at: https://www.researchgate.net/publication/344853679

\section{Micro/macro-scale robotic approach for middle ear surgery}

Article in IEEE Transactions on Medical Robotics and Bionics · October 2020

DOI: 10.1109/TMRB.2020.3032456

CITATIONS

READS

3 authors, including:

Q9. Tamadazte Brahim

Institut FEMTO-ST

96 PUBLICATIONS 785 CITATIONS

SEE PROFILE

Some of the authors of this publication are also working on these related projects:

Project Microrobotics View project

Project NEMRO: Impact of Smell Deficiency on Neurodegenerative Diseases View project 


\title{
Micro/macro-scale robotic approach for middle ear surgery
}

\author{
Jae-Hun $\mathrm{So}^{1}$, Brahim Tamadazte ${ }^{1,2}$, and Jérôme Szewczyk ${ }^{1}$.
}

\begin{abstract}
This paper deals with the development of a robotic solution for middle ear surgery. The surgical procedure consists of the resection of pathological tissues that occurs inside the middle ear cavity known as cholesteatoma. The current surgical procedure is performed manually, is invasive and does not guarantee an exhaustive cholesteatoma tissues removal leading to reoccurrence in $25-30 \%$ cases. To remedy this, we developed a new surgical protocol that combines a robotic solution and original surgical instruments. On one side, it consists of a redundant seven degrees of freedom (DoFs) robotic arm extended with a 2 DoFs flexible fibroscope (emulating a future microrobot under development). On the other side, different control architectures are proposed to achieve the surgical procedure. One control mode is based on the teleoperation of whole developed system using a joystick such as an Omni device. The other mode combines comanipulation of the 7 DoFs robotic arm using an embedded force/torque sensor and teleoperation of the remaining 2 DoFs fibroscope using a lab-made in-hand joystick. The proposed materials and methods were validated experimentally using a robotic setup. The obtained results using the different control schemes are discussed.
\end{abstract}

Index Terms-Medical robotics, teleoperation, comanipulation, middle ear surgery.

\section{INTRODUCTION}

C HOLESTEATOMA is an abnormal skin growth that occurs in the middle ear cavity. It is usually due to ventilation problem where dead skin cells cannot be ejected out of the ear. Gradually, the cholesteatoma expands in the middle ear, filling in the empty cavity around the ossicles and then eroding the bones themselves (ossicles and mastoid). Cholesteatoma is often infected and results in chronically draining ears. It also results in hearing loss and may even spread through the skullbase into the brain. It was reported that one case per 10,000 citizens occurs every year in European countries. Currently, the most effective treatment for cholesteatoma is to surgically resect or ablate the infected tissues [1]. This procedure consists of addressing the middle ear cavity invaded by cholesteatoma simultaneously through the external ear canal and through a large mastoidectomy [2]. Mastoidectomy is a procedure which consists of drilling the mastoid in order to get direct access to the middle ear cavity. The surgeon uses this made access to insert the dedicated surgical tools which are rigid and straightline miniature instruments. Moreover, this direct access is necessary to visualize the surgical site when using an external view (an optical stereo-microscope).

\footnotetext{
${ }^{1}$ Authors are with Sorbonne Université, CNRS UMR 7222, INSERM U1150, ISIR, F-75005, Paris, France. sodisir.upmc. fr

${ }^{2}$ Author is with FEMTO-ST Institute, Univ. Bourgogne Franche-Comté, CNRS, Besançon, France.
}

It can be highlighted that the current surgery protocol is invasive and requires high-expertise of the surgeon. Also, the access and the visualization of the entire middle-ear cavity through the incision hole is very challenging. Consequently, a perfect and exhaustive ablation of the tissue is not guaranteed and leads, in $25-30 \%$ cases, to a second surgery, 6 to 18 months after the first intervention due to the growth of the residual cholesteatoma. Hence, there is a high demand for improvement of current surgical procedures towards less invasive approaches; i.e., reducing the incision hole and avoiding a second surgical intervention by ensuring an exhaustive resection of the infected tissues.

Using a flexible miniature and actuated tool passing through a small incision hole will allow accessing the entire epitympanum cavity where cholesteatoma develops while reducing the invasiveness of the traditional surgical procedure. This alternative could definitely help the clinician to exhaustively remove the cholesteatoma. Indeed, mounting the actuated tool on a robotic arm would increase both the dexterity and the accuracy of the surgical system.

In a recent survey, Dahroug et al. [3], reports different innovations and developments carried out as part of middle ear surgery including robotic holders, surgical tools, imaging systems, as well as the requirements for an optimal system and surgical protocol for middle ear surgery. In fact, several robots have been designed for middle ear microsurgery to bring back sufficient accuracy, dexterity and ergonomy to the physician. They are mainly dedicated to cochlear implantation [4], tympanic membrane grafts or ossicular chain replacement with a prosthesis [5] which requires high accuracy and reproducibility. However, all these solutions are based on rigid tools and do not include any dexterous instrument for intracorporeal fine movement execution. Other works highlight the feasibility of robot-assisted ear surgery such as robotassisted mastoidectomy [6] and tool-guidance [7] which is not sufficient for the cholesteatoma treatment in which surgeons mainly suffer from a lack of dexterity and accuracy in tooltip positioning. The main contributions of this paper are the design of a redundant macro/micro-scale robotic structure with embedded teleoperation interface as well as the development of two control modes for robotic middle ear surgery execution. The control modes are 1) an end-frame teleoperation of the whole kinematic structure with an intuitive and transparent management of the fulcrum effect and 2) a combination of a comanipulation of the macro-scale robot and an in-hand teleoperation of the micro-scale tool. 

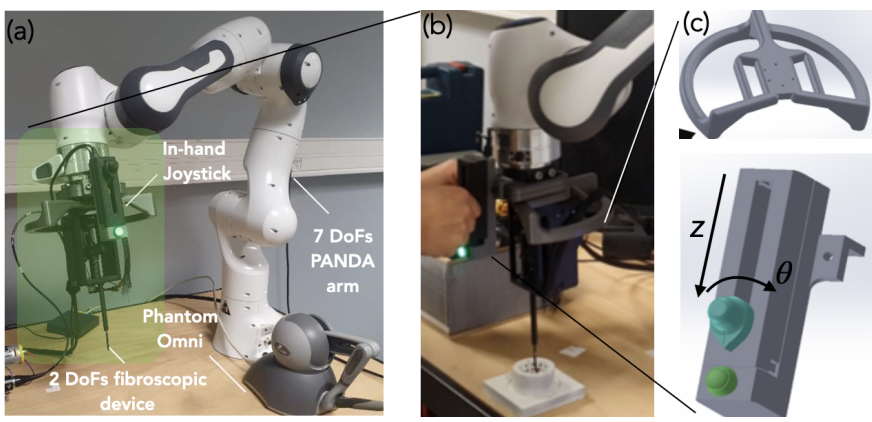

Fig. 1. Overview of the designed robotic setup for middle ear surgery. (a) the whole system, (b) a zoomed view on the distal part of the robot including the micro-scale 2 DoFs system, and (c) the surgeon-robot interfaces used for both comanipulation and in-hand teleoperation of the instrument

\section{MATERIALS}

As discussed in the introduction, the cholesteatoma surgery requires accuracy, ergonomics and intuitiveness, especially for junior surgeon. To meet these challenges, we propose an original approach by combining a macro-scale system (robotic arm) and a micro-scale system (flexible microrobotic endoscope for cholesteatoma resection).

The macro-scale system is comprised of a 7 DoFs collaborative robotic arm PANDA from Franka Emika ${ }^{\circledR}$ in which is attached at the distal part, a $6 \mathrm{DoFs}$ force/torque sensor of type of ATI MINI-40, as well as an ergonomic lab-made handle in the form of a half steering wheel (Fig. 1(a)). Additionally, a 2 DoFs microrobotic sub-system is attached to the distal part of the PANDA robotic arm. Thus, the obtained full system is a redundant 9 DoFs robot which can be controlled either in comanipulation manner or in teleoperation mode using a Sensable Phantom Omni. Note that the development of a specific flexible microrobot devoted to the cholesteromatous tissue resection is currently in progress, so we have used a miniature actuated 2 DoFs OLYMPUS IF2D5-12 fibroscope to mimic the future microrobotic system. This fibroscopic device can be controlled via a lab-made miniature and lightweight joystick which is attached to the PANDA end-effector (Fig. 1(c)). In this work, we use only the mechanical features of the fibroscope and not the provided images.

\section{MODELLING AND CONTROL}

The outlined specifications for the surgical system are based on ergonomics, easy-to-use and accuracy required by the achievement of the surgical procedure. In order to control the whole redundant robotic system (macro and micro-scale robots), we propose two control modes; i.e., a teleoperation controller and combination of comanipulation of the macroscale robot with an in-hand teleoperation for the micro-scale system.

Controlling a 9 DoFs robot in the context of the minimally invasive surgery is challenging. The classical approach consists of tele-operating the system at its effector level while relying on an automatic management of joint redundancies to cope with the kinematic constraints at the point of insertion in the body [8]. However, this approach is complex and requires a perfect positioning of the robot within the task-space (patient's middle ear). Simpler solutions are based on a two-handed tele-operation of the robot in the task-space [9] or on a special management of the movements of its end-effector [10]. Nevertheless, these approaches lead to an increase of the cognitive workload and operation time. This is why, in this paper, we evaluate a new approach using a comanipulation scheme for the macro-scale sub-system and an in-hand joystick for the teleoperation of the micro-scale one. We compare this original solution to the classical end-effector teleoperation solution. The kinematical feature of is described below on the Figure 2 and was used to derive the system's kinematic model.

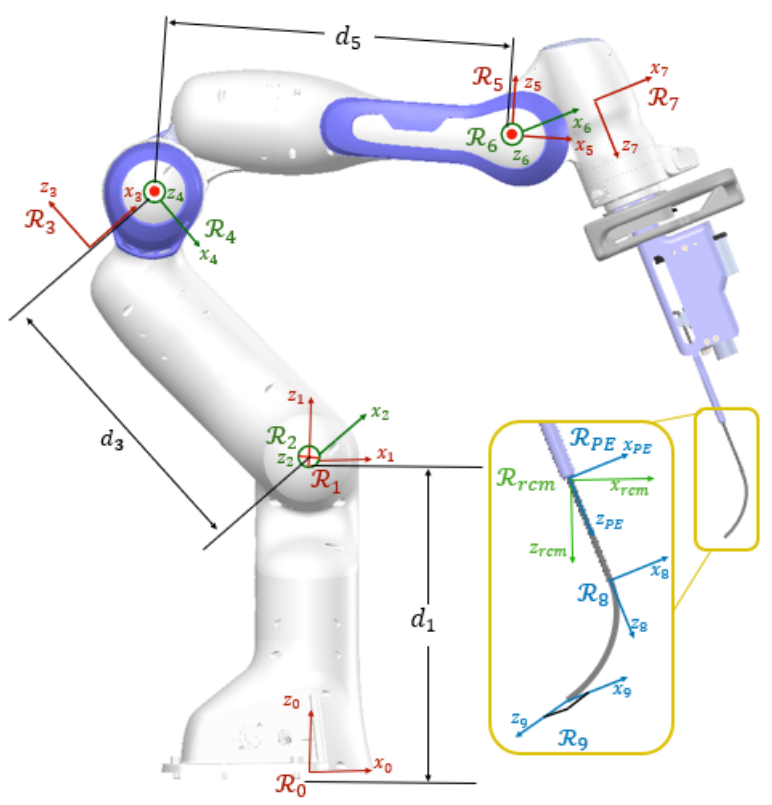

Fig. 2. Kinematic model of the developed system with the associated frames.

\section{A. End-frame Teleoperation}

Teleoperation mode is based on a position-based controller allowing the interpretation of the local pose of the Phantom Omni end-effector as the desired pose of the micro-scale system tip. In addition, as the micro-scale tool enters inside the middle ear cavity through the incision hole,its motion is kinematically constrained in a fulcrum effect manner at a point located at the origin of a fixed frame $\mathcal{R}_{r c m}$ as illustrated in Fig. 2.

In order to simplify the computational process, the movements of the PANDA's end-effector frame $\mathcal{R}_{P E}$ will be expressed in RCM (Remote Center Motion) frame $\mathcal{R}_{r c m}$ instead of its original base frame $\mathcal{R}_{0}$. Moreover, in order to comply with the kinematic constraints at the ear entrance point, we chose to make the origin of $\mathcal{R}_{P E}$ and $\mathcal{R}_{r c m}$ to always coincide during the task. Thus, only 3 rotations between frame $\mathcal{R}_{r c m}$ and $\mathcal{R}_{P E}$ expressed by $q_{x}, q_{y}, q_{z}$ are possible.

Consequently, as depicted in Fig. 2, as the kinematic chain between $\mathcal{R}_{P E}$ and the system terminal frame $\mathcal{R}_{9}$ is completed by the 2 DoFs of the actuated fibroscope. The system provides to the operator 5 intracorporeal controllable DoFs; 
i.e., $\mathbf{Q}=\left(q_{x}, q_{y}, q_{z}, q_{8}, q_{9}\right)^{\top}$. Assuming that the distal tool rotation around its own axis is of no importance for the task (e.g. laser shooting), these 5 intracorporeal DoFs are sufficient for the tool-tip motion control.

The corresponding joint-space velocities are $\dot{\mathbf{Q}}=$ $\left(\dot{q}_{x}, \dot{q}_{y}, \dot{q}_{z}, \dot{q}_{8}, \dot{q}_{9}\right)^{\top}$ and the task-space velocity vector is expressed by $\dot{\mathbf{X}}_{r c m}=(\dot{x}, \dot{y}, \dot{z}, \dot{\alpha}, \dot{\beta}, \dot{\gamma})^{\top}$. The relationship between task-space velocities and the joint-space ones can be expressed, using a Jacobian $\mathbf{J}_{r c m} \in \mathbb{R}^{6 \times 5}$ defined as follows:

$$
\dot{\mathbf{X}}_{r c m}=\mathbf{J}_{r c m} \dot{\mathbf{Q}}
$$

For control purposes, it is required to invert relation (1) in order to get the joint-space velocities from the desired tool-tip ones. Indeed, $\mathbf{J}_{r c m}$ being a $6 \times 5$ matrix at this stage, we can reduce it to a $5 \times 5$ format by ignoring the tool rotation around its own axis; i.e., along $z_{9}$. Leaving this rotation uncontrolled has no consequence on the task execution. Accordingly, the corresponding joystick own rotation on the master side will not be accounted for. Thus, we obtain:

$$
\dot{\mathbf{Q}}=\widetilde{\mathbf{J}}_{9}^{-1} \dot{\mathbf{X}}_{9}
$$

where $\dot{\mathbf{X}}_{9}=\left(\dot{x}_{9}, \dot{y}_{9}, \dot{z}_{9}, \dot{\alpha}_{9}, \dot{\beta}_{9}\right)^{\top}$ and $\widetilde{\mathbf{J}}_{9}^{-1} \in \mathbb{R}^{5 \times 5}$ is the inverse matrix of the new simplified $\mathbf{J}_{r c m}$ matrix. Based on this, the overall teleoperation scheme can be depicted as on Fig. 3.

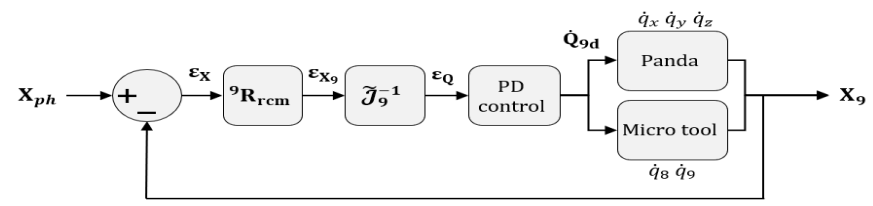

Fig. 3. Control loop of the tip-frame teleoperation with RCM simplification.

The Phantom Omni's end-effector pose $\mathbf{X}_{p h}$ is the desired set-point for the micro-scale tool's end-effector pose. The difference of the two poses is expressed in $\mathcal{R}_{9}$ using the rotation matrix ${ }^{9} R_{\text {rcm }}$. Thus, the task-space error $\epsilon_{X_{9}}$ is converted into a joint-space error noted $\epsilon_{Q}$ using (2). This error is then minimized through a proportional-derivative (PD) controller giving the desired joint-space velocities as $\dot{\mathbf{Q}}_{9_{d}}=\left(\dot{q}_{x}, \dot{q}_{y}, \dot{q}_{z}, \dot{q}_{8}, \dot{q}_{9}\right)^{\top}$ where $\dot{q}_{x}, \dot{q}_{y}, \dot{q}_{z}$ are the rotational velocities moving the PANDA arm around the fixed origin of $\mathcal{R}_{r c m}$ and $\dot{q}_{8}$ and $\dot{q}_{9}$ the joint velocities relative to the microscale tool.

\section{B. Macro-comanipulation and Micro-teleoperation}

The second control scheme is based on an intuitive association of a comanipulation scheme for the macro-scale subsystem and an in-hand teleoperation for the micro-scale one as shown in Fig. 4. Therefore, the surgeon can achieve the approaching task; i.e., bring the robot from any position to a desired one corresponding to that of the surgical site. The surgeon can also control simultaneously the micro-scale tool with the other hand by the in-hand lab-made joystick. Note that is this scenario, this control strategy does not require any registration effort.

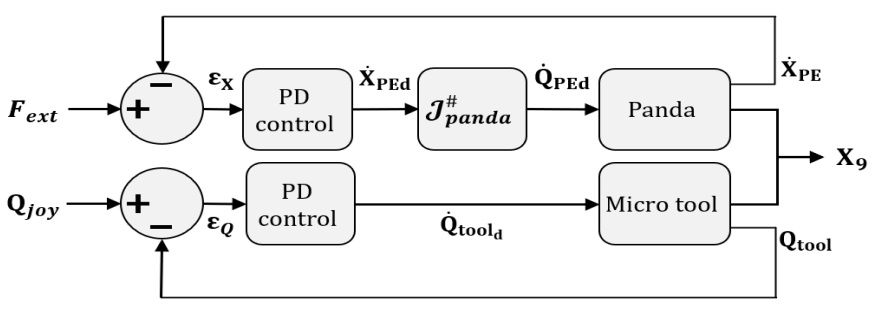

Fig. 4. Control scheme modelling the association of a macro-comanipulation and micro-teleoperation control modes.

The implementation of this controller is relatively undemanding. The force and torque noted $F_{\text {ext }}$ provided by the embedded force sensor on the PANDA end-effector frame $\mathcal{R}_{P E}$ is converted into desired operational velocities that serve as the inputs of a classical PD loop (upper path in Fig. 4). Furthermore, to control the 2 DoFs micro-scale device, a simple joint-position controller is carried out based on a desired joint position specified using the embedded microjoystick (lower path in Fig. 4). Note that because it is mounted on the moving PANDA's end-effector, the micro-joystick base frame always remains aligned with the micro-tool base frame thus making the tool-tip teleoperation intuitive.

\section{VALIDATION AND RESULTS}

\section{A. Validation Scenario}

To replicate the surgical protocol (removing residual cholesteatoma tissues) and reproduce, at a scale up factor 2 , the middle ear cavity environment (access constraints and limited visibility), we designed the test-bench shown in Fig. 5. Inside the designed cavity, we added 5 targets (red dots) which represent possible residual cholesteatoma which has to be pointed by the micro-scale system for resection/ablation; e.g., by laser shots. The carried-out validation scenario consists of positioning as accurately as possible the fibroscope tip in front of and orthogonal to the simulated residual cholesteatoma. The micro-scale tool positioning task is performed first using the end-frame teleoperation method and then using the hybrid comanipulation and in-hand teleoperation approach.

Furthermore, the visualization of the surgical site is provided thanks to an external camera simulating an endoscopic system which would be introduced through the ear canal during the targeted surgical protocol. Therefore, in the validation scenario discussed here, the view provided by the fibroscope is not used by the operator during the task. An example of the view provided by the external camera is shown in Fig. 5 .

\section{B. Results Analysis}

The experimental scenario was carried out by three subjects. The size of the target points is $3 \mathrm{~mm}$ of diameter similar to residual cholesteatoma tissues. Before experiment, the 3D poses (position and orientation) of each target are recorded. During experiment, when the subject judges that the target is reached, the reference poses are compared to the actual 3D pose of micro-scale tool-tip.

The obtained results are depicted in Fig. 6 where we represented the $3 \mathrm{D}$ poses achieved using both proposed control 


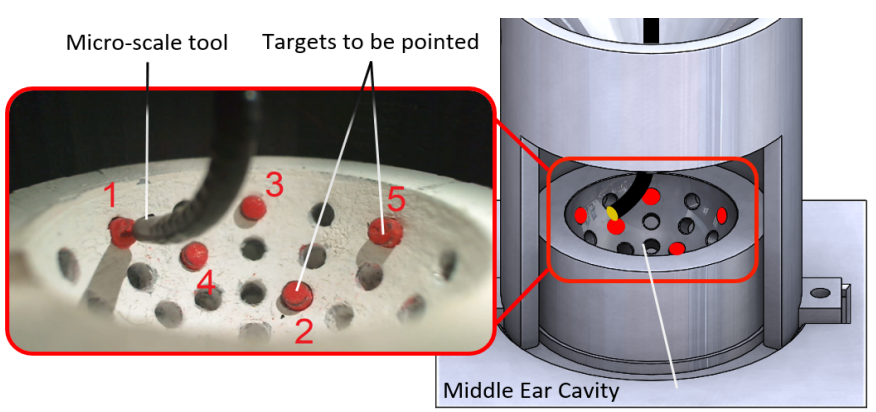

Fig. 5. Representation of the targets to be pointed at by the robotic system miming residual cholesteatoma.

modes. The corresponding numerical values are summarized in Table I and compared to those obtained when the operation is performed manually. The positioning error is separated into $\bar{e}_{a x i}$, the axial error along the $z$ axis of the fibroscope device and $\bar{e}_{l a t}$, the lateral error corresponding to the planar positioning error within the $x-y$ plane. Note that in the targeted surgical protocol, , the surgeon will have to position the surgical tool-tip in front of the residual cholesteatoma (in $x-y$ plane) without any obligation to touch it.

The mean linear error $\bar{e}_{\text {lat }}$ for all control modes are below $2 \mathrm{~mm}$ which is in phase with the predefined medical requirements. Moreover, the mean angular error $e_{r}$ is below 0.2 degree for both control modes but slightly high for manual handling with 0.73 degree. Throughout this preliminary study, robot assisted task execution demonstrates a better performance than manual handling as the total mean error are smaller for both modes. Otherwise, the comanipulation with in-hand teleoperation appears slightly more precise comparing to the end-frame teleoperation method as the standard deviation for total lateral error is greater for teleoperation case. Concerning the time required to achieve the pointing task, it is estimated to 142 seconds for the teleoperation mode and 184 seconds for the comanipulation and in-hand teleoperation.

TABLE I

MEAN LINEAR LATERAL ERROR $\bar{e}_{\text {lat }}$ AND AXIAL $\bar{e}_{a x i}$ ERROR (MM), MEAN ANGULAR ERROR $\bar{e}_{r}$ (DEGREE), AVERAGE OF THE FIVE TESTS $\mu$ AND THE CORRESPONDING STANDARD DEVIATIONS E(.).

\begin{tabular}{l|ccc|ccc|ccc|}
\cline { 2 - 10 } $\mathrm{N}^{\circ}$ & \multicolumn{3}{|c|}{ Comanipulation } & \multicolumn{3}{c|}{ Teleoperation } & \multicolumn{3}{c|}{ Manual } \\
\cline { 2 - 10 } 1 & $\bar{e}_{\text {lat }}$ & $\bar{e}_{a x i}$ & $\bar{e}_{r}$ & $\bar{e}_{\text {lat }}$ & $\bar{e}_{\text {axi }}$ & $\bar{e}_{r}$ & $\bar{e}_{\text {lat }}$ & $\bar{e}_{a x i}$ & $\bar{e}_{r}$ \\
\cline { 2 - 10 } 2 & 1.76 & 0.66 & 0.26 & 3.16 & 2.00 & 0.18 & 2.5 & 0.7 & 0.42 \\
3 & 2.03 & 1.40 & 0.10 & 0.78 & 1.48 & 0.21 & 3.1 & 0.9 & 1.29 \\
4 & 1.21 & 2.52 & 0.31 & 0.66 & 0.35 & 0.37 & 1.2 & 1.3 & 0.40 \\
5 & 1.61 & 1.60 & 0.14 & 1.30 & 0.55 & 0.11 & 1.4 & 0.6 & 0.68 \\
$\mu$ & 1.67 & 0.71 & 0.30 & 1.25 & 0.94 & 0.41 & 0.9 & 1.1 & 0.83 \\
$\mathrm{E}()$. & $\mathbf{1 . 6 6}$ & 1.38 & $\mathbf{0 . 2 2}$ & $\mathbf{1 . 4 3}$ & 1.06 & $\mathbf{0 . 2 6}$ & 1.84 & 0.88 & 0.73 \\
\cline { 2 - 10 } & 0.50 & 0.93 & 0.08 & 1.01 & 1.86 & 0.11 & 0.84 & 1.04 & 0.32 \\
\hline
\end{tabular}

Although the obtained performance meet the surgical requirements in this scenario, significant improvements are expected thanks to the future miniature robot (concentric tube robot) and a narrower view of the surgical site (endoscopic system introduced in the middle ear cavity through the ear canal). In fact, the fibroscope system that mimic the microrobotic tool, showed substantial limitations in terms of resolution and repeatability due to its cable-based actuation.
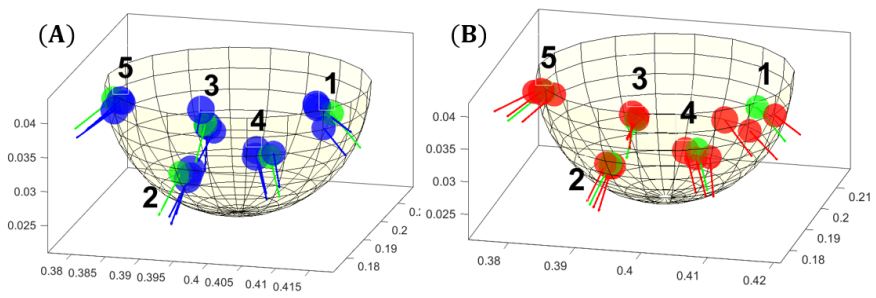

Fig. 6. Results of the pointing task. (a) comanipulation method (green spheres the desired poses and the blue ones the final poses of the micro-scale tip), and (b) end-frame teleoperation (green spheres are the desired poses and the red ones the final poses of the micro-scale tip).

\section{CONCLUSION AND DISCUSSIONS}

In this paper, the development of an original redundant robotic system for minimally invasive surgery is presented. The proposed architecture is obtained by a combination of a 7 DoFs macro-scale robotic arm with a 2 DoFs microscale tool. To perform the surgical task which consists of the resection/ablation of pathological tissues; e.g., using laser shooting, we proposed two different control modes : an endframe teleoperation scheme and a comanipulation approach for the macro-scale system associated to an in-hand teleoperation of the micro-scale tool. Both the proposed system and the control methods were successfully tested experimentally. Future work will focus on a deeper evaluation of the system and its controllers by surgeons in clinical conditions. Also, the micro-tool which is currently based on a 2 DoFs classical fibroscope system will be replaced by a flexible surgical tool based on a continuum micro-robot mechanism which is under development.

\section{ACKNOWLEDGMENT}

This work is supported by French ANR $\mu$ RoCS Project no ANR-17-CE19-0005-04 and by the Investissement d'Avenir program (labex CAMI) under reference ANR-11-LABX-0004.

\section{REFERENCES}

[1] E. L. Derlacki et al., "Lx congenital cholesteatoma of the middle ear and mastoid," Ann. of Oto., Rhin. \& Lary., vol. 74, pp. 706-727, 1965.

[2] P. Bordure, et al. Chirurgie otologique et otoneurologique. Elsevier Masson, 2005.

[3] B. Dahroug, et al., "Review on otological robotic systems: Toward microrobot-assisted cholesteatoma surgery," IEEE Rev. in Biomed. Eng., vol. 11, pp. 125-142, 2018.

[4] B. Bell, et al. "In vitro accuracy evaluation of image-guided robot system for direct cochlear access," Oto. \& Neuro., vol. 34, pp. 1284-1290, 2013.

[5] M. Miroir, and al., "Design, kinematic optimization, and evaluation of a teleoperated system for middle ear microsurgery," The Scient. World J., 2012.

[6] M. H. Yoo, et al., "A cadaver study of mastoidectomy using an image-guided human-robot collaborative control system," Lary. Invest. Oto., vol. 2, no. 5, pp. 208-214, 2017.

[7] K. Entsfellner, et al., "A modular micro-macro robot system for instrument guiding in middle ear surgery," IEEE Int. Conf. on Rob. and Biom., pp. 374-379, 2015.

[8] A. Simorov, et al., "Review of surgical robotics user interface: what is the best way to control robotic surgery?" Surg. Endo., vol. 26, pp. 2117-25, 2012.

[9] K. Entsfellner, et al., "Micro-Macro Telemanipulator for Middle-Ear Microsurgery," in German Conf. on Robo., 2012, pp. 395-398.

[10] A. Tobergte, et al."The sigma.7 haptic interface for MiroSurge: A new bi-manual surgical console," IEEE Int. Cong. on Intel. Rob. and Sys., pp. 3023-3030, 2011. 